\title{
Caso clínico: \\ Síndrome de Brugada manejo anestésico
}

\section{Case report: Anesthetic management of Brugada Syndrome}

María Belén Chango Salas ${ }^{1}$ Daysi Noemí Segovia Bustamante ${ }^{1}$, Jenny Mabel Vanegas Mendieta ${ }^{1}$

\begin{abstract}
Brugada Syndrome is a rare genetic condition that affects cardiac ion channels and predisposes patients to ventricular arrhythmias and sudden death[1],[2]. It could be responsible for between 20 and $60 \%$ of the idiopathic ventricular fibrillations that occur during the anesthetic act[3]. Most patients have uncomplicated courses with anesthesia, surgery, and invasive procedures. However, there is a risk of worsening ventricular arrhythmias and ST elevation due to perioperative medications, surgical aggression, electrolyte disturbances, fever, autonomic nervous system tone, as well as other disturbances[2],[3]. Given the increasing Number of patients with inherited conduction disorders presented for non-cardiac surgery who are at risk for sudden cardiac death, safe anesthetic management depends on a detailed knowledge of these conditions[5].
\end{abstract}

\section{RESUMEN}

El síndrome de Brugada es una condición genética rara, afecta los canales iónicos cardíacos y predispone a los pacientes a arritmias ventriculares y muerte súbita[1],[2]. Pudiera ser responsable de entre el 20 y el $60 \%$ de las fibrilaciones ventriculares idiopáticas, $4-12 \%$ de todas las muertes súbitas y aproximadamente el $20 \%$ de las muertes en pacientes con un corazón estructuralmente sano[3]. La mayoría de los pacientes tienen cursos sin complicaciones con anestesia, cirugía y procedimientos invasivos. Sin embargo, existe el riesgo de empeoramiento de las arritmias ventriculares y elevación del ST debido a los medicamentos peri operatorios, agresión quirúrgica, alteraciones electrolíticas, fiebre, tono del sistema nervioso autónomo, así como otras per-

\section{Key words:}

Anesthesia, arrhythmias,

Brugada syndrome

\section{Palabras clave:}

Anestesia, arritmias, perioperatorio, Síndrome de Brugada

Médico Posgradista de Anestesiología. Hospital Carlos Andrade Marín, Universidad San Francisco de Quito.

Fecha de recepción: 07 de julio de 2018

Fecha de aceptación: 11 de septiembre de 2018

\section{ORCID}

https://orcid.org/0000-0002-0746-8867

Correspondencia:

Jenny Mabel Vanegas Mendieta

E-mail: mabe_8717@hotmail.com 
turbaciones[2],[4]. Dado el creciente número de pacientes con trastornos de la conducción heredadas que se presentan para cirugía no cardiaca que están en riesgo de muerte súbita cardiaca, el manejo anestésico seguro depende de un conocimiento detallado de estas condiciones[5].

\section{Introducción}

- I síndrome de Brugada se incluye entre las ca- nalopatías, trastornos eléctricos primarios que característicamente no asocian cardiopatía estructural concomitante[1].

La prevalencia se sitúa en torno a 5/10.000 habitantes[1],[4]. La edad de diagnóstico en promedio es 40-50 años, el 80\% de pacientes afectos son hombres[2].

Descrito por primera vez en 1992 como un cuadro consistente en muerte súbita en pacientes portadores de un patrón electrocardiográfico de bloqueo de rama derecha con un supra desnivel del ST persistente en las derivaciones V1 a V2-V3 característico, con un intervalo Q-T normal[3].

Estudios genéticos han determinado su herencia autosómica dominante con penetrancia variable, afecta los canales de sodio causado por mutaciones del gen SCN5A que codifica la subunidad alfa de los canales cardiacos de sodio y como consecuencia se produce una reducción de las corrientes de entrada de este ion durante el potencial de acción sobre todo a nivel epicárdico[3]. La repolarización no homogénea resultante es la causa de arritmias ventriculares[1].

Se han descrito tres patrones electrocardiográfi- cos distintos (Figura 1)[1]

a) Patrón tipo I: elevación descendente del segmento $\mathrm{ST} \geq 2 \mathrm{~mm}$ en más de una derivación precordial derecha (V1-V3), seguida de ondas T negativas.

b) Patrón tipo II: caracterizado por elevación del segmento ST $\geq 2 \mathrm{~mm}$ en precordiales derechas seguida de ondas T positivas o isobifásicas, lo que confiere al electrocardiograma un aspecto de silla de montar

c) Patrón tipo III: definido como cualquiera de los dos anteriores si la elevación del segmento ST es $\leq$ $1 \mathrm{~mm}$.

Aunque los tres patrones pueden observarse en el síndrome de Brugada incluso en el mismo paciente en momentos diferentes, sólo el tipo I se considera diagnóstico de la enfermedad[1].

Datos de estudios multicéntricos confirman que entre los pacientes que han sufrido una muerte cardíaca súbita recuperada, el $62 \%$ presenta una nueva arritmia en un periodo medio de 54 meses[6]. Ello significa que estos pacientes deben protegerse con desfibrilador automático implantable como prevención secundaria[1],[2],[3].

Se decidió la presentación del caso por la poca prevalencia de la enfermedad y lo complejo del manejo anestésico en relación a conocimiento de fármacos

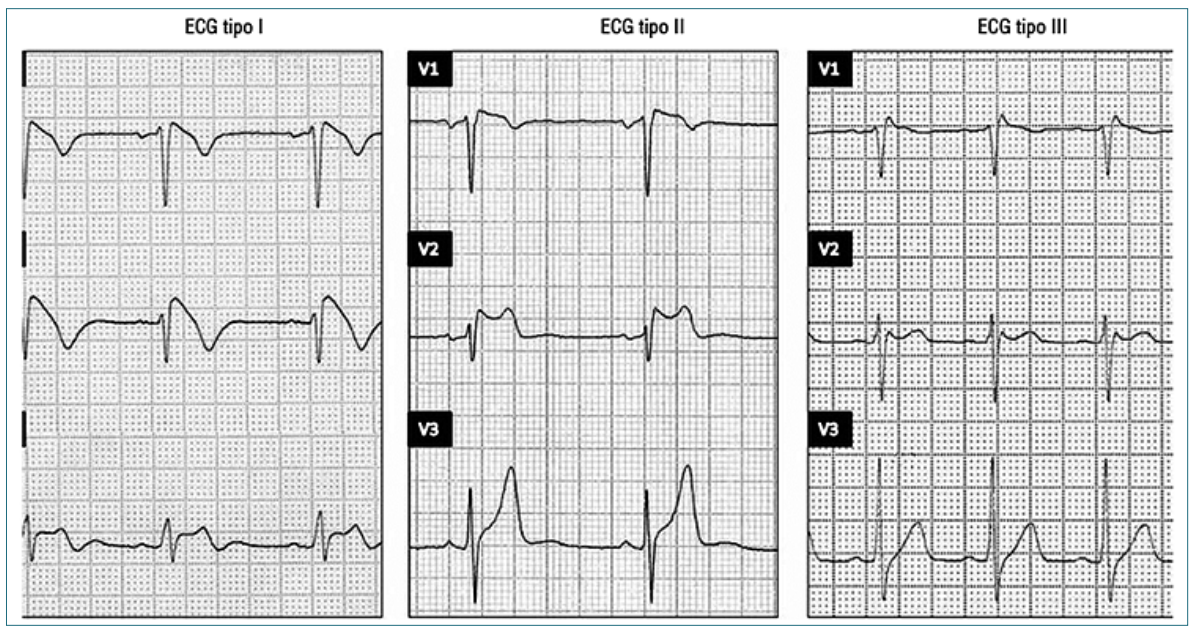

Figura 1. Patrones electrocardiográficos de síndrome de Brugada[1]. 
y consideraciones específicas que pueden ser desencadenantes de arritmias fatales.

\section{Caso clínico}

Paciente masculino de 45 años de edad sin antecedentes clínicos ni quirúrgicos, ingresó al servicio de emergencias por pérdida súbita de conciencia, en monitorización se evidenció fibrilación ventricular, realizaron desfibrilación externa automática, posterior a lo cual presentó ritmo nodal, aseguraron vía aérea y fue ingresado a unidad de cuidados intensivos. A las 24 horas presentó nuevo episodio de fibrilación ventricular el cual revirtió a desfibrilación externa, electrocardiograma en donde se evidenció ritmo nodal, bloqueo de rama derecha, QTC 440 mseg.

Estudios paraclínicos dentro de parámetros normales, ecocardiograma sin reporte de lesiones estructurales.

El servicio de cardiología estableció como diagnóstico un patrón de Brugada.

Una vez estabilizado se planificó colocación de cardiodesfibrilador automático implantable bajo anestesia general. Previa monitorización básica no invasiva, accesos vasculares de alto flujo y asegurando la disponibilidad de un desfibrilador externo.

Manejo anestésico con infusión de Remifentanilo y Sevofluorane. Durante procedimiento mantuvo estabilidad macrodinámica (TAM 70), sin requerimiento de soporte vasopresor, frecuencia cardíaca 55-60 latidos por minuto, monitorización electrocardiográfica continua sin hallazgos anormales.

Finalizado el procedimiento quirúrgico se induce fibrilación ventricular para comprobar funcionamiento del cardiodesfibrilador automático implantado.

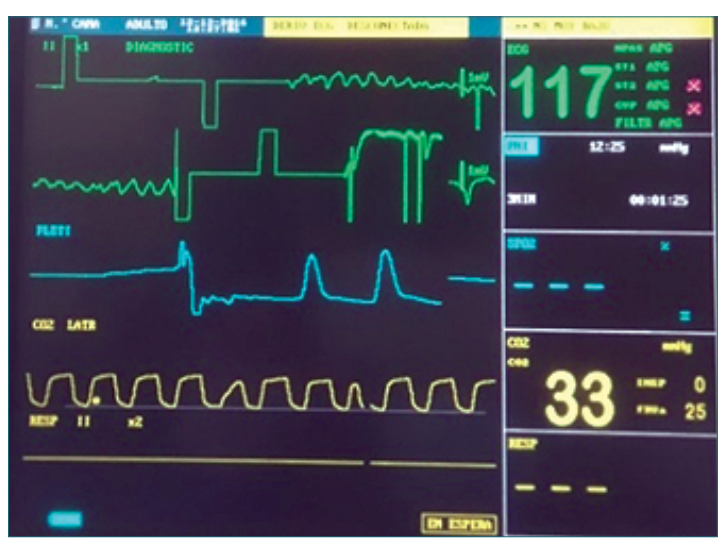

Figura 2. Fibrilación ventricular provocada.

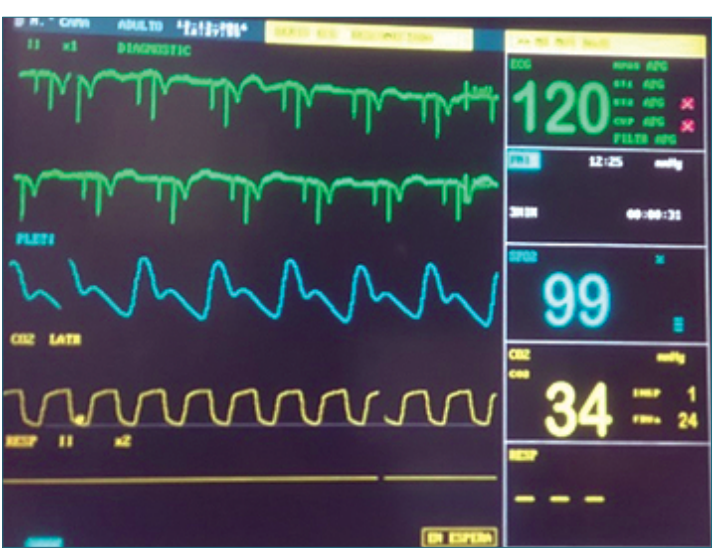

Figura 3. Funcionamiento de cardiodesfibrilador automático implantado.

Extubación previo reversión de bloqueo neuromuscular con atropina y neostigmina e integridad de reflejos protectores de vía aérea.

Paciente egresó a unidad de cuidados post-anestésicos hemodinámicamente estable.

Permaneció hospitalizado durante 48 horas posterior al procedimiento sin presentar ninguna complicación, fue dado de alta para seguimiento por consulta externa.

\section{Discusión}

La principal preocupación en los pacientes con Síndrome de Brugada es la susceptibilidad a taquiarritmias ventriculares y muerte cardíaca súbita[7]. Ciertas condiciones y factores pueden empeorar el patrón electrocardiográfico.

Es importante para el anestesiólogo familiarizarse con el síndrome para ofrecer un manejo perioperatorio seguro al paciente que lo padece[4].

\section{Consideraciones no farmacológicas}

En el período preoperatorio asegurar correcciones de electrolitos: hipo e hiperkalemia e hipercalcemia ya que pueden empeorar la elevación del segmento ST[4].

Durante el procedimiento quirúrgico asegurar un plano anestésico adecuado debido a que el incremento de la actividad simpática como lo ocurrido durante la incisión, la orointubación y extubación podrían ser desencadenantes de arritmias fatales, evitar cambios abruptos de posición y procurar normoter- 
$\operatorname{mia}[4],[6],[7]$.

Los aumentos repentinos en el tono parasimpático (aspiración traqueal, insuflación peritoneal y retracción intestinal) constituyen principales desencadenantes de fibrilación ventricular[6],[1].

Pacientes con diagnóstico establecido con y sin cardiodesfibrilador implantado deben tener parches para desfibrilador colocados y asegurar un desfibrilador externo cercano al campo quirúrgico[7].

Si el paciente cuenta con un desfibrilador automático interno desactivarlo antes del evento quirúrgi$\mathrm{co}[8]$.

Evitar el sistema de cauterio monopolar, empleando idealmente sólo el bipolar, por el potencial de generar arritmias en el transoperatorio[8].

\section{Consideraciones farmcológicas}

Los fármacos que deben ser evitados en el síndrome de Brugada, incluyen anti arrítmicos clase IA y clase IC, antidepresivos tricíclicos, litio, oxcarbazepina, acetilcolina, ergonovina, cocaína, marihuana, alcohol, aunque hay reportes de casos aislados con uso de beta bloqueadores e inhibidores de canales de calcio, la lista es extensa y una revisión ampliada se encuentra disponible en www.BrugadaDrugs.org[5], [6].

Consideraciones con fármacos de uso común en Anestesia.

\section{Agentes intravenosos}

Propofol: su uso es controversial al alterar la función de los canales iónicos de sodio y calcio. Está en la lista de drogas a evitar con recomendación lla. Los reportes de casos han demostrado su empleo en monodosis para inducción sin alteraciones, sin embargo se ha descrito patrones electrocardiográficos de Brugada con su infusión continua[7],[8],[9].

Etomidato: en el estudio de Kloesel se describió elevación del segmento S-T autolimitada posterior a su empleo[9].

Opioides: por su efecto bradicardizante pueden facilitar la aparición de complicaciones, preferentemente usar los de acción corta o ultracorta[7],[9]. Estudios han demostrado que el tramal y fentanilo bloquean con mas potencia los canales de sodio, siendo el tramal documentado como causa de alteraciones electrocardiográficas con patrón de Brugada[7],[9].

\section{Anestésicos inhalatorios}

El uso de agentes volátiles puede prolongar el intervalo Q-T, el óxido nitroso, desfluorane, enfluo- rane, sevofluorane pueden ser empleados satisfactoriamente para mantenimiento en anestesia general. Sevofluorane ha sido sugerido como el anestésico inhalatorio de lección debido a que no tiene efecto sobre la longitud del intervalo QT[2],[5],[7].

\section{Bloqueantes neuromusculares}

Succinilcolina: uso controversial. En dosis para inducción anestésica en secuencia rápida puede inducir cambios en la membrana celular y mayor riesgo al repetir la dosis[2],[9].

\section{Agentes de reversión}

Neostigmina: por estímulo parasimpático puede generar cambios del S-T, su uso es controvertido y se considera la administración de manera lenta[2],[9].

Sugammadex: puede ser utilizado con seguridad en estos pacientes[10].

\section{Anestésicos locales}

Pueden desencadenar cambios electrocardiográficos al bloquear los canales de sodio. Se han descrito estudios que avalan la seguridad en anestesia neuroaxial con lidocaína, mepivacina, bupivacaina, ropivacaína, sin embargo, se considera menos aconsejable el uso de Bupivacaina ya que su isómero $R$ bloquea ávidamente los canales de sodio y se disocia muy lentamente, convirtiéndola en el fármaco con mayor potencial proarrítmico y cardiotóxico[3],[7]

\section{Agentes vasopresores}

Puede usarse con seguridad efedrina o fenilefrina, los betabloqueantes y agonistas alfa deben ser usados con precaución, pueden causar elevación del segmento ST y enmascarar un patrón electrocardiográfico de Síndrome de Brugada[4].

En el caso presentado se administró anestesia general con agente halogenado e infusión de opioide intravenoso, considerando cada uno de los fármacos que pueden ser causantes de arritmias fatales se realizó una revisión sobre la seguridad de la administración de Propofol en monodosis y el uso de anestésicos locales (lidocaína endovenosa), siendo descrita como una práctica segura[7]

\section{Conclusiones}

Las arritmias graves pueden ser desencadenadas por varios factores presentes durante los procedimientos quirúrgicos, que ponen en riesgo la seguridad del paciente.

Es mandatorio para el Anestesiólogo conocer cri- 
terios diagnósticos de esta entidad, así como también los fármacos que deben ser evitados por su potencial implicación como desencadenantes de arritmias fatales.

\section{Reconocimientos}

Asistencia para estudio: ninguno.

Apoyo financiero y patrocinio: ninguno.

Conflictos de interés: ninguno.

\section{Referencias}

1. Benito B, Brugada J, Brugada R. Sindrome de Brugada. Rev Esp Cardiol. (2009);62(11):129731. Disponible en: http://www. revespcardiol.org/es/sindromebrugada/articulo/13142828/ https://doi.org/10.1016/S03008932(09)73082-9.

2. Kloesel B, Ackerman MJ, Sprung J, Narr BJ, Weingarten TN. Anesthetic management of patients with Brugada syndrome: a case series and literature review. Canadian Journal of Anesthesia/Journal canadien d'anesthésie. Springer Science and Business Media LLC; 2011 Jun 23;58(9):824-36. Disponible en: http://dx.doi.org/10.1007/ s12630-011-9546-y

3. Sorajja D. Ramakrishna H, Poterack $A$. Brugada syndrome and its relevance in the perioperative period. Annals of Cardiac Anaesthesia (2015) Vol 18(3). Disponible en: http://www. annals.in/temp/AnnCardAaesth183403-4717389_130613. pdf

4. Smith D, Martz D. Brugada Syndrome: A Review of Perioperative Management For Anesthesiologists. Anesthesiology news (2015) 69-72. Disponible en: https://www.anesthesiologynews.com/Review-Articles/ Article/10-15/Brugada-Syndrome-A-Review-of-PerioperativeManagement-For-Anesthesiologists/33891/ses=ogst

5. Vhora J. Rajagopalan J. Update on the Diagnosis and Management of Brugada Syndrome. Heart, Lung and Circulation (2015) 24, 1141-1148. Disponible en: https://www. heartlungcirc.org/article/S14439506(15)01330-X/pdf

6. Zúñiga S, Dávila Cabo de Villa E, Quesada L. Síndrome de Brugada y anestesia regional. Presentación de un caso. Medisur. Cienfuegos (2016) vol.14 (6) Disponible en: http://scielo.sld. cu/pdf/ms/v14n6/ms13614.pdf

7. Flamée $P$, De Asmundis $C$, Bhutia JT, Conte G, Beckers S. Safe single-dose administration of propofol in patients with established Brugada syndrome: a retrospective database analysis. Pacing Clin Electrophysiol. (2013) 36(12):1516-21. Disponible en: https://www.ncbi.nlm.nih.gov/ pubmed/23952345 https://doi. org/10.1111/pace.12246.

8. López-Jiménez F, Mondragón M. Síndrome de Brugada y anestesia. (2008) Vol. 31. (1).5562. Disponible en: http://www. medigraphic.com/pdfs/rma/cma2008/cma081i.pdf

9. Abad A, Espinoza A, Ripolles J. Síndrome de Brugada y Anestesia: Manejo Anestésico. Asociación Anestesia Reanimación España. Revista Electrónica de AnestesiaR (2016) vol. 8 (3). Disponible en: http://revistaanestesiar.org/index.php/rear/article/ view/98/282

10. Biricik E, Hatipoglu Z, Küçükbingöz Ç. Sugammadex in a Patient with Brugada Syndrome. Turk J Anaesthesiol Reanim. 2016 Apr;44(2):99-101. https://doi. org/10.5152/TJAR.2016.60973 PMID:27366567 